\title{
Photopolarographic Behavior of Inorganic Depolarizers
}

\author{
R. A. Durst* and J. K. Taylor
}

(July 21, 1965)

\begin{abstract}
Nickel(II), cobalt(II), and uranium(VI) were studied polarographically to determine the effect of uv-visible irradiation on their reduction characteristics. Photoeffects were observed in all cases. Two mechanisms are proposed for the nickel photokinetic prewave, and the complex photoeffects observed with cobalt and uranium are described. Proposals are made for further studies to clarify and elucidate the photochemical reactions and to apply this technique in the study of the activated states of ions prior to reduction.
\end{abstract}

Key words: Cobalt(II), depolarizers, nickel(II), photoactivation, photochemical effects, photopolarographic effects, polarographic measurements, radiation effects, uranium (VI).

\section{Introduction}

Although the effects of uv-visible irradiation on organic compounds have been studied for some time $[1],{ }^{1}$ the application of photopolarography to inorganic systems has received little attention. This paper describes some previously unreported phenomena observed during the photopolarographic reduction of nickel(II), cobalt(II), and uranyl ions. The data obtained to date are still mostly qualitative and descriptive with only tentative explanations and interpretations given. The present preliminary work has been concerned primarily with surveying possible systems for future detailed study.

This investigation was stimulated by a research proposal for the study of the activation overpotential of irreversible redox systems presented at the Massachusetts Institute of Technology in February 1962 [2] in which it was proposed to activate certain irreversible couples by irradiation with uv-visible light and determine the magnitude of the activation by the shift in the halfwave potential of the irreversible electrode reaction. It was presumed that a study of this type in which the activation energies are evaluated could lead to an understanding of the structural changes taking place in the course of the electrochemical reaction.

Vlček [3] has suggested that since the activation energy is a direct measure of the energy necessary for the formation of the transition state, by comparing the values of the activation energy with known constants of the particular substance, such as the energies of the spectral transitions, structural changes may be estimated and an insight into the mechanism of the whole reaction can be obtained.

Before the electron transfer process can occur, the electronic states of the depolarizer and of the electrode must be nearly equal and, in addition, the electron is always accepted by the lowest unoccupied (or singly

*Chemistry Department, Boston College. Chestnut Hill, Mas

Figures in brackets indicate the literature references at the end of this paper. occupied) orbital in the depolarizing particle. However, when there is no low-lying orbital available, electronic rearrangement must take place to create a vacant orbital into which subsequently an electron can be accepted and retained [4]. Since the electron transfer itself is always very rapid, the cause of irreversibility in these cases is that these particles cannot accept or retain the electrons without first undergoing some change in their electronic structure.

In this investigation, an attempt was made to produce these necessary electronic changes by irradiation with high-intensity, uv-visible light. The promotion of the electrons to higher orbitals by the absorption of radiation should make lower lying orbitals available for the electron transfer. This means that the energy requirement for the formation of the transition state and consequently the electron transfer should be reduced by an amount equivalent to the energy of the spectral transition. Similarly, the electrode potential necessary for the electron transfer should be reduced but in a more complex manner since both the transfer coefficient and the heterogeneous rate constant must be considered.

In attempting to apply polarography to this type of study, two serious problems immediately arise. One is obtaining the required resolution in the half-wave potentials of the waves for the reduction of the activated and ground-state ions, and the other is measuring the small photokinetic current produced by the activation. Since photoactivation is basically a very inefficient process, the concentration of activated ions in the vicinity of the electrode will be very low, necessitating an extremely sensitive polarograph. Fortunately, the activated species will be reduced at potentials more anodic than the ground-state ions so that the interference from the much larger concentration of unactivated ions will not be as serious. Furthermore, the steady-state concentration of the activated species will be proportional to the intensity of the radiation although limits in this respect are set by available equipment and the necessity of avoiding heating effects in the solution. In addition, the steady-state con- 
centration of the activated ions, and consequently the photokinetic current observed, will depend on the rate of decay or half-life of the activated ions.

From the above discussion, it is expected that the shift of the half-wave potential for an irreversible reduction to more anodic values during irradiation should result in a photokinetic prewave; the degree of anodic shift being dependent on the wavelength (energy) of absorbed radiation and the transfer coefficient, the wave height (photokinetic current) dependent on the concentration of the ground-state ions, radiation intensity, and decay rate of the activated ions. Conversely, no prewave should occur upon irradiation of an inorganic depolarizer which is reversibly reduced under normal conditions.

\section{Apparatus}

The polarograph used in this study was an electronic-scan, controlled-potential type incorporating several features $[5,6]$ advantageous in achieving the resolution and sensitivity required to detect and measure the small photokinetic prewave. These features include a derivative mode of operation which permits the separation of the prewave from the main reduction wave, and parallel-T RC filters which remove the charging current oscillations to further increase the resolution of the derivative polarograms. In addition, the use of a three-electrode system consisting of the dropping mercury electrode (DME), an asbestos-fiber saturated calomel electrode (SCE), and a platinum working electrode eliminates the need for iR drop corrections. The peak follower was also used to eliminate oscillations for the recording of maximum current polarograms with an insignificant amount of damping.

The polarographic cell consists of a single-compartment rectangular Corex cell $(5 \times 5 \times 2 \mathrm{~cm})$ with optically flat surfaces covered by an aluminum foil light shield with circular openings $1 \mathrm{~cm}$ in diameter in the center of the front and rear surfaces. An opaque plastic top has openings for the three electrodes and the nitrogen deaeration inlet and outlet tubes. These components are arranged in such a way that only the mercury drop is in the excitation light beam.

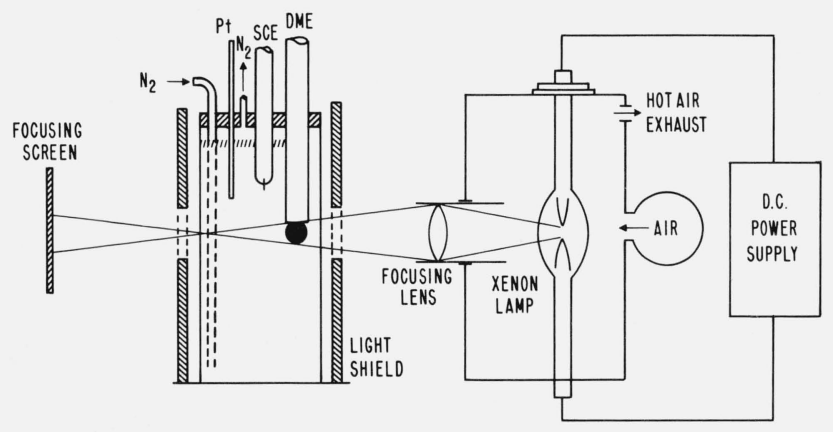

Figure 1. Polarographic cell and xenon lamp excitation source.
The excitation source is a quartz high-pressure xenon lamp which produces an intense uv-visible continuum in the range from 0.25 to 0.75 microns and is powered by a d-c power supply. The lamp is mounted in a special chamber fitted with a focusing lens and forced-air cooling device (fig. 1). The light from the xenon lamp is focused by positioning the lens so that the light beam is almost completely blocked by the mercury drop at maximum size. This is accomplished by observing the drop image on the focusing screen and adjusting accordingly.

\section{Results}

\subsection{Nickel(II)}

The first inorganic depolarizer to be studied by photopolarography was the aquo nickel(II) ion. The reduction of nickel from noncomplexing media is irreversible, as indicated by the half-wave potential being more than $0.5 \mathrm{~V}$ more negative than would be predicted from the reversible potential of the $\mathrm{Ni}^{++}-\mathrm{Ni}$ couple [7]. In order to produce a measurable steadystate concentration of the activated nickel(II), it was necessary to use a relatively high concentration of the nickel salt. Therefore, $0.50 M$ reagent-grade nickel sulfate solutions were used. Since this work was primarily concerned with observing photoeffects and not with quantitative results, no supporting electrolyte was used. Also, inasmuch as the prewave of interest is a kinetic wave rather than a diffusion wave, it is unnecessary to eliminate the migration current. Actually, the migration of nickel ions to the electrode is desirable insofar as enhancement of the photokinetic wave is concerned. In order to avoid any side photoreactions, the use of a maximum suppressor was also omitted. In order to obtain the optimum resolution and sensitivity, derivative polarograms were taken using the 1-2-4-10 filters. The polarograms are shown in figure 2.

Due to the high concentration and sensitivity, the nickel(II) derivative wave begins at about $-0.65 \mathrm{~V}$ versus SCE, and the half-wave potential occurs at approximately $-1.1 \mathrm{~V}$. With irradiation, a photokinetic wave precedes this main nickel reduction wave. The separation is not great enough to allow the prewave to develop fully, but using derivative polarography the half-wave potential is easily obtained. During the continued irradiation, the half-wave potential of the photokinetic prewave shifts between -0.70 and $-0.75 \mathrm{~V}$ versus SCE in an irregular manner, and the peak current fluctuates between 2 and $6 \mu \mathrm{A}$. The heating effect in the solution and especially around the dropping mercury electrode (DME) is small as evidenced by the very small anodic shift of the rising portion of the ground-state nickel wave.

Since the principal absorption band for nickel (II) occurs at $3950 \mathrm{~A}$, the amount of energy absorbed by the $\mathrm{Ni}\left(\mathrm{H}_{2} \mathrm{O}\right)_{6}^{++}$ions is equal to $72.2 \mathrm{kcal} / \mathrm{mol}$ (energy $=N h c(\lambda)$ or 3.13 electron volts. According to the ligand field theory [8]. this corresponds to a $d$-d transition in $\mathrm{Ni}(\mathrm{II})$ which has eight $3 d$ electrons. In octahedral coordination, as in the case of $\mathrm{Ni}\left(\mathrm{H}_{2} \mathrm{O}\right)_{6}^{++}$, the ground state electronic configuration of $\mathrm{Ni}(\mathrm{II})$ is ${ }^{3} \mathrm{~A}_{2, g}\left(t_{2 g}\right)^{6}\left(e_{g}\right)^{2}$. The absorption of energy at $3950 \AA$ 


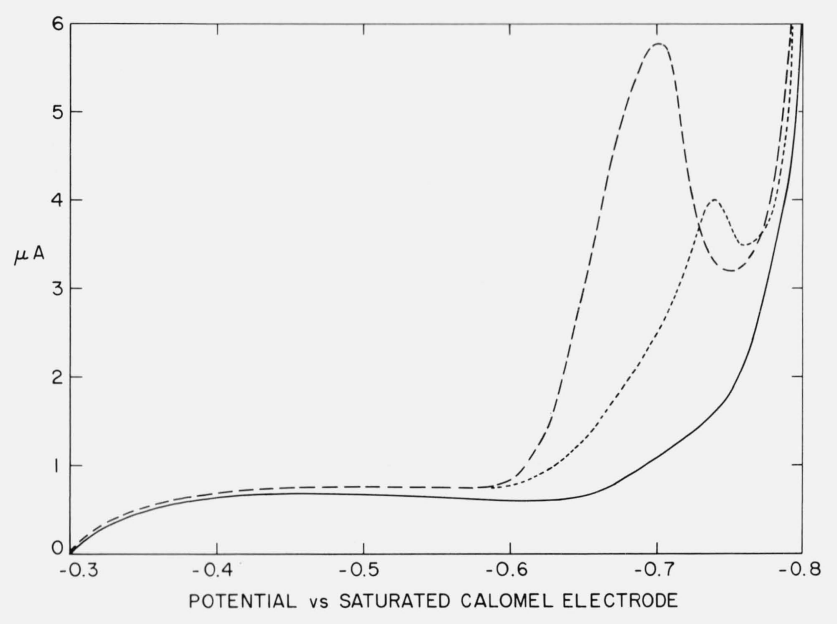

Figure 2. Polarographic reduction waves of nickel(II). Solution, $0.50 M$ nickel sulfate: scan rate, $0.1 \mathrm{~V} / \mathrm{min}$ : derivative mode of operation: $-2-4-10$ filters.

No irradiation.

With irradiation (anodic extreme).

With irradiation (intermediate).

$\left(25,300 \mathrm{~cm}^{-1}\right)$ corresponds to the transition: ${ }^{3} \mathrm{~A}_{2 g}$ $\rightarrow{ }^{3} \mathrm{~T}_{1 y}(\mathrm{P})$, or more simply, a transition from the $\left(t_{2 g}\right)^{6}\left(e_{g}\right)^{2}$ configuration to the $\left(t_{2 g}\right)^{4}\left(e_{g}\right)^{4}$ configuration. Since $e_{g}$ electrons are antibonding, i.e.. their presence tends to weaken and thus lengthen the metal-ligand bonds $\left(\mathrm{Ni}^{++}-\mathrm{H}_{2} \mathrm{O}\right)$ while $t_{2 g}$ electrons are nonbonding and have little effect on the metal-ligand bond lengths $[9]$, this transition whould result in a weakening of the aquonickel complex. As it is necessary to remove the complexing water molecules during the reduction of nickel to the metal form (zero oxidation state), this weakening of the nickel-water bonds could result in the smaller energy requirement for the reduction as is observed in figure 2 .

Another possible explanation of the activation of the $\mathrm{Ni}(\mathrm{II})$ as evidenced by its more reversible reduction during irradiation is by the method originally proposed. That is, when electrons are raised to higher energy orbitals $\left(e_{g}\right)$, lower energy orbitals $\left(t_{2 g}\right)$ are made available for the acceptance of the reducing electrons. Either one or both of these mechanisms could explain the increased reversibility and, for the present, this problem cannot be unequivocally answered.

\subsection{Cobalt(II)}

Hexaquo cobaltous ions are irreversibly reduced in noncomplexing media at a half-wave potential of about - 1.4 V versus SCE. In cobaltous chloride solutions containing no supporting electrolyte, the cobalt is present as the pink $\mathrm{Co}\left(\mathrm{H}_{2} \mathrm{O}\right)_{6}^{++}$ion which is reduced at a potential several tenths of a volt more negative than would be predicted from the reversible potential of the $\mathrm{Co}^{++}-\mathrm{Co}$ couple [10]. To be able to detect any activation effects, the relatively high concentration of $0.10 M$ reagent-grade cobaltous chloride was used in the polarographic solutions. Again, it was unnecessary and undesirable to use a supporting electrolyte and

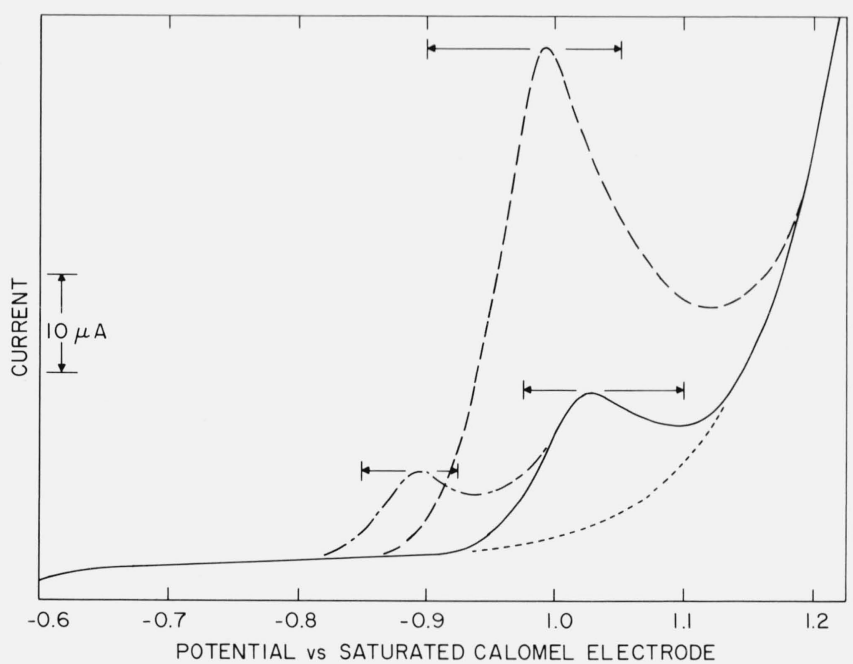

Figure 3. Polarographic reduction waves of cobalt(II).

Solution, $0.10 \mathrm{M}$ cobaltous chloride: scan rate, $0.1 \mathrm{~V} / \mathrm{min}$ : 1-2-4-10 filter With or without irradiation, deaerated, $p \mathrm{H}<6.5$. No irradiation, $p \mathrm{H} \geqslant 7.0$, not deaerated (Brdička hydrolysis wave). With irradiation, not deaerated, $p \mathrm{H} \geqslant 6.5$.

No irradiation, trace of $\mathrm{H}_{2} \mathrm{O}, 2$ added.

maximum suppressor for the reasons previously stated in the discussion of the $\mathrm{Ni}(\mathrm{II})$ reduction.

In figure 3 , the preliminary results of the Co(II) study are summarized. Due to the large separation of the prewave from the main reduction wave, it was unnecessary to use the derivative mode of operation of the polarograph to obtain resolution of the waves. The peaks on these waves are, therefore, maxima and not to be confused with derivative peaks which would indicate the half-wave potential. The polarograms were taken using the 1-2-4-10 filters to eliminate drop oscillations and give smooth average current traces. From the figure, it is seen that the Co(II) wave in solutions of $p \mathrm{H}$ less than 6.5 begins at about $-1.0 \mathrm{~V}$ versus SCE at the cobalt concentration and instrument sensitivity used. This is the typical, irreversible reduction wave of $\mathrm{Co}\left(\mathrm{H}_{2} \mathrm{O}\right)_{6}^{++}$as would be expected. Upon irradiation, no observable changes are produced in this wave which would indicate either the activation effect does not occur with cobalt or the rate of decay of the activated species is too rapid to allow the buildup of a detectable concentration.

In neutral or slightly basic solutions, however, a prewave does occur at potentials between -1.0 and - 1.1 V versus SCE (as indicated by the arrows extending from the peak of the wave). This wave occurs in the absence of irradiation and is most certainly the hydrolysis wave reported by Brdička [11]. The prewave described by Brdička is attributed to reduction of the hydrolysis product of the hexaquo cobaltous ions:

$$
\left[\mathrm{Co}\left(\mathrm{H}_{2} \mathrm{O}\right)_{6}\right]^{++} \rightleftharpoons\left[\mathrm{Co}\left(\mathrm{H}_{2} \mathrm{O}\right)_{5} \mathrm{OH}\right]^{+}+\mathrm{H}^{+} .
$$

The hydrolysis equilibrium is established at a rate slow enough for each ion to be reduced at its own potential. The absence of the prewave in acid solution is caused by the suppression of hydrolysis according to the above equilibrium. 
With irradiation, a very large prewave occurs in solutions of $p \mathrm{H}$ equal to or greater than 6.5 at potentials between -0.9 and $-1.05 \mathrm{~V}$ versus SCE. Although the prewave produced on irradiation generally occurs at potentials about $0.05 \mathrm{~V}$ more anodic than the hydrolysis wave, the overlap of potentials is considerable, and one is tempted to interpret the waves as being identical except in their magnitude. Although Brdicka noted that heating increased the size of the hydrolysis wave, the position of the rising portion of the main Co(II) wave is not shifted anodically and thus precludes heating as a significant effect.

For reasons still not understood, prewaves could not be produced, either with or without irradiation, when the solutions were deaerated. Indeed, it appeared that the size of the wave produced with irradiation increased with increasing oxygen concentration. This observation led to the conjecture that the irradiation prewave might be due wholly or in part to the production of hydrogen peroxide by the action of uv radiation on the oxygen and water. This hypothesis was tested by the addition of trace amounts of $\mathrm{H}_{2} \mathrm{O}_{2}$ to the polarographic solutions. At the lowest concentrations, a wave of the type shown in figure 3 was obtained at potentials of about $-0.9 \mathrm{~V}$ versus SCE. In general, the hydrogen peroxide wave occurred at potentials more anodic than the irradiation prewave although there was considerable overlap. In addition to the $\mathrm{H}_{2} \mathrm{O}_{2}$ wave, the hydrolysis wave appeared at potentials between the peroxide and hexaquo Co(II) waves. Since the prewave caused by irradiation arises at potentials between the $\mathrm{H}_{2} \mathrm{O}_{2}$ and hydrolysis waves, another possible interpretation of the wave produced during irradiation is a merging of these two waves. This seems unlikely, however, since one would expect to see a double-peaked prewave at some point of development during irradiation if this were the case. At higher hydrogen peroxide concentra-

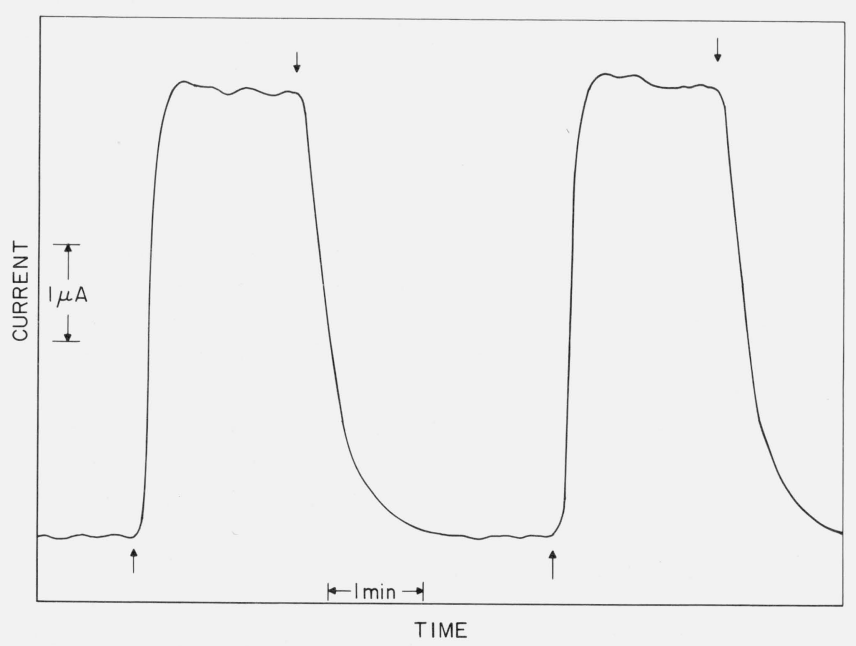

Figure 4. Photoenhancement and decay of the $C o(I I)$ prewave. Solution, $0.10 M$ cobaltous chloride, $p \mathrm{H}=6.7$, potential at peak of photoinduced wave, half-life of photoactivated species $\simeq 12$ seconds.

$\uparrow$ Xenon lamp-ON.

$\downarrow$ Xenon lamp-OFF tions, it appears that the $\mathrm{Co}(\mathrm{II})$ catalyzes the reduction of $\mathrm{H}_{2} \mathrm{O}_{2}$ as reported by Strnad [12] and the oxidation of mercury. Moreover, at these concentrations of $\mathrm{H}_{2} \mathrm{O}_{2}$, there is considerable distortion of the polarogram in the region of the prewaves and of the main Co(II) reduction wave. Since such high hydrogen peroxide concentrations do not arise by irradation, these effects may be disregarded and further discussion of this type of interfering reaction omitted.

Although these complicating side reactions prevent the use of $\mathrm{Co}$ (II) in the manner originally intended, i.e., in an investigation of changes in the electronic configuration during electrochemical reduction, the very large enchancement of the prewave during irradiation stimulated one additional study. When the potential is set at the peak of the photoinduced prewave and the irradiation applied periodically, the current variations clearly show the production and decay of the photoactivated species. Figure 4 shows a typical current versus time curve for the prewave during alternate periods of irradiation and darkness. The activation process is very rapid, reaching equilibrium in approximately $\frac{1}{2} \mathrm{~min}$. The decay of the activated species is considerably slower, being on the order of $1 \frac{1}{2}$ min for equilibrium and exhibiting a half-life of about 12 sec. The peak current trace shown in this figure was obtained by connecting the maximum current oscillations of the polarograms recorded without damping or filtering. The current fluctuations during the period of irradiation are probably due to variations in the intensity of the xenon lamp.

Except for the rather interesting occurrences noted above in the reduction of $\mathrm{Co}(\mathrm{II})$, the results obtained do not warrant further study of cobalt under the original project. Although solutions of cobaltous chloride show definite photoeffects, these seem not to be of the electronic configuration type desired in this investigation, but rather due to photochemically induced side reactions which complicate the interpretation of the results and thereby prevent the use of cobalt in this type of study.

\subsection{Uranium(VI)}

The use of the $\mathrm{UO}_{2}^{++}$ion in a study of photopolarographic behavior is also complicated by side reactions. However, the well-known behavior of uranyl ion as a photosensitizer makes this ion an interesting prospect even though it is reversibly reduced and should not exhibit an activation prewave. The polarographic reduction of uranyl ions may take place in as many as four steps corresponding to reduction to the (V), (IV), (III), and metal amalgam states [13]. However, at all acidities, the reduction corresponding to the first wave is

$$
\mathrm{UO}_{2}^{++}+e^{-} \rightarrow \mathrm{UO}_{2}^{+},
$$

although in nearly neutral solutions, the hydrolysis of 
the uranyl ion

$$
\mathrm{UO}_{2}^{++}+\mathrm{H}_{2} \mathrm{O} \rightarrow \mathrm{UO}_{2} \mathrm{OH}^{+}+\mathrm{H}^{+}
$$

causes a decrease in the magnitude of the first wave [14]. The hydrolysis product, $\mathrm{UO}_{2} \mathrm{OH}^{+}$, is irreversibly reduced at about $-0.6 \mathrm{~V}$ versus $\mathrm{SCE}$ and overlaps the $\mathrm{U}(\mathrm{V}) \rightarrow \mathrm{U}(\mathrm{IV})$ wave.

A summary of typical results is given in figure 5 . The uranyl reduction derivative peak occurs at approximately $-0.24 \mathrm{~V}$ versus SCE and exhibits a slight maximum (as indicated by the dip in the curve between -0.3 and $-0.4 \mathrm{~V}$ ). During irradiation, there is not the slightest trace of a prewave which is in agreement with what would be expected for a reversible reduction, but a series of "postwaves" do occur. On a normal polarogram, these postwaves would appear as a series of maxima or undulations on the upper part of the wave and on the plateau. Even more curiously, these undulations (except for the first) seem to continuously shift in an anodic direction during continued irradiation. The first peak at $-0.28 \mathrm{~V}$ is the most prominent and stable of these current fluctuations. Over an irradiation time of more than an hour, this peak shifts less than $10 \mathrm{mV}$ either anodically or cathodically, although its height varies by about $15 \mu \mathrm{A}$ (fig. 5 shows the maximum height of all the peaks). The second peak (at $-0.32 \mathrm{~V}$ in fig. 5) has been followed by successive polarograms over a period of an hour and was found to shift $60 \mathrm{mV}$ anodically. It has been difficult to follow the remainder of the peaks from one polarogram to the next since the peak heights vary considerably, and an individual peak usually cannot be unequivocally distinguished on successive polarograms. However, in some cases this was achieved, and these smaller peaks seem to follow the anodic-shift trend.

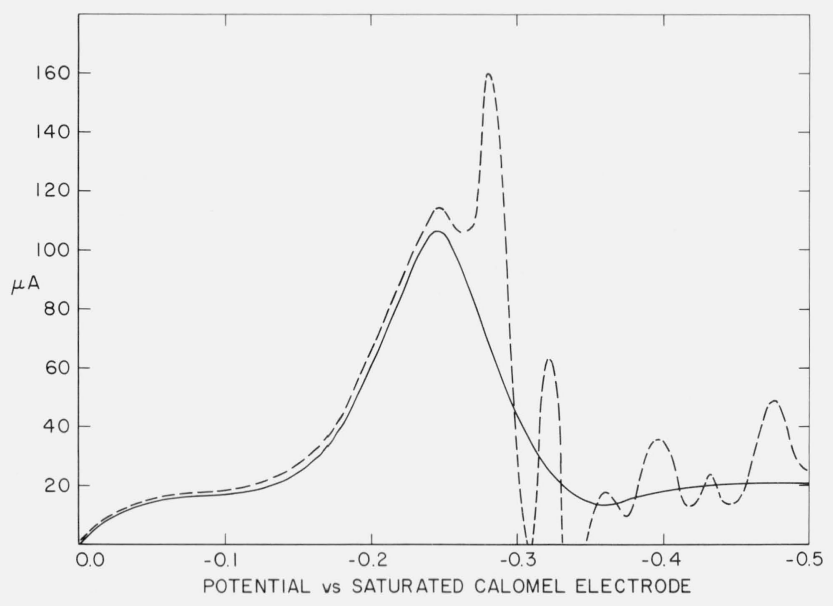

Figure 5. Polarographic reduction waves of $\mathrm{UO}_{2}^{++}$.

Solution, $0.020 M \mathrm{UO}_{2} \mathrm{SO}_{4}+0.1 M \mathrm{Na}_{2} \mathrm{SO}_{4}$ : scan rate, $0.1 \mathrm{~V} / \mathrm{min}$ : derivative mode of operation: $1-2-4-10$ filters

No irradiation.

...... With irradiation.
Although a rigorous interpretation cannot be made at the present time for these fluctuations in the uranyl reduction current, two observations would seem to eliminate hydrolysis as the cause of the observed photoeffect. The first is the fact that the main uranyl peak height does not decrease during irradiation as would be expected if hydrolysis occurred. And second, polarograms recorded to $-0.8 \mathrm{~V}$ versus $\mathrm{SCE}$ do not show any indication of the hydrolysis wave which should occur at $-0.6 \mathrm{~V}$. All that is certain at the present time is that a definite photoeffect does occur with the uranyl ion, and an explanation of its cause must await further polarographic studies.

\section{Discussion and Future Considerations}

Since this study was intended as a preliminary survey of possible systems for further detailed research, many points remain to be clarified and evaluated. For example, the effect of different wavelengths of light on the activation process could provide information on the actual energies required for maximum activation. This would further elucidate the electronic transitions taking place during the electrochemical reduction. Eventually it may be possible to apply mathematical treatments such as those of Koutecky [15] and Koutecky and Brdička [16] for the kinetics of irreversible reductions and reductions involving the generation of the depolarizer in the vicinity of the electrode, and also correlate these with the observed photokinetic currents. In addition, it is presently unknown what effect the irradiation has on the mercury drop itself. Since mercury is a known photosensitizer, the results observed may be due to a transfer of energy from the mercury rather than direct absorption of energy by the ions in solution. Perhaps the spectral studies or studies with other types of electrodes will provide the answers.

Although the use of cobalt(II) and uranium (VI) can be eliminated from consideration in the present investigation due to their complex photoeffects, their study has indicated additional areas in which photopolarography may be applied to induce and analyze different types of photoreactions which have heretofore gone unreported. Certainly phenomena of this type deserve more attention as they may lead to greater understanding of the reactions of simple and complex ions in excited states.

From the reported observations, it appears that the aquo nickel(II) ion is the most likely prospect for further research into the photoactivation of an irreversibly reduced species. The two mechanisms proposed for the apparent increased reversibility of nickel during irradiation gives some indication of the profound changes that may be induced by this technique. Since nickel is but one of the many species which are irreversibly reduced, inorganic photopolarography could have wide application in studying and elucidating these reductions, and it is to be hoped that these initial results will stimulate further research into these phenomena. 


\section{References}

[1] H. Berg and H. Schweiss, Nature 191, 1270 (1961).

[2] R. A. Durst, Photo-voltammetric studies of the activation overpotential of irreversible systems, Analytical Chemistry Proposition I, M.I.T., Feb. 5, 1962 (unpublished report).

[3] A. A. Vlček, Collection Czechoslov. Chem. Communs. 24, 3538 (1959).

[4] A. A. Vlček, Disc. Faraday Soc. 26, 164 (1958).

[5] M. T. Kelley, H. C. Jones, and D. J. Fisher, Anal. Chem. 31, 1475 (1959).

[6] M. T. Kelley, D. J. Fisher, and H. C. Jones, Anal. Chem. 32, $1262(1960)$.

[7] I. M. Kolthoff and J. J. Lingane, Polarography, II, p. 486 (Interscience Publishers, New York, 1952).

[8] C. J. Ballhausen, Introduction to Ligand Field Theory, p. 261 (McGraw-Hill Book Co., New York, N.Y., 1962).
[9] F. A. Cotton, J. Chem. Ed. 41, 466 (1964).

[10] R. Brdička, Collection Czechoslov. Chem. Communs. 2, 489 (1930).

[11] Ibid. 3, 396 (1931)

[12] F. Strnad, Collection Czechoslov. Chem. Communs. 11, 391 (1939).

[13] I. M. Kolthoff and P. J. Elving, Treatise on Analytical Chemistry, p. 115, P. II, 9, (Interscience Pub., New York, 1962).

[14] W. E. Harris and I. M. Kolthoff, J. Amer. Chem. Soc. 69, 446 (1947).

[15] J. Koutecky, Collection Czechoslov. Chem. Communs. 18, 597 (1953).

[16] J. Koutecky and R. Brdička, Collection Czechoslov. Chem. Communs. 12, 337 (1947).

(Paper 69A6-372) 